\title{
Correspondence
}

\section{TRICHLORETHYLENE POISONING MIMICKING MULTIPLE SCLEROSIS}

\section{To the Editor}

The spectrum of trichlorethylene (TCE) toxicity is broad and was recently highlighted by two patients referred to our Multiple Sclerosis (MS) Clinic.

\section{Case 1}

Five minutes after exposure to a spill of TCE, this technician noticed an unpleasant taste, vertigo and chest tightness (three hours duration). Numbness of the left face and leg (L5 dermatome) developed within seven days and persisted. One year later, after using TCE for thirty minutes, he became aware of an unpleasant odour followed by lightheadedness, dyspnea, and nausea which persisted for two hours. The following day numbness of the left C5-6 dermatome (duration 3 weeks) and dorsum of the left foot (permanent) occurred. A neurologist found no abnormalities on examination. A CT scan, EEG, and two series of visual, auditory, and somatosensory evoked potentials done at six-monthly intervals were normal. MS was suspected and he was referred to the University Hospital MS Clinic. His history was otherwise unremarkable and his examination was normal. A magnetic resonance imaging (MRI) study of the head was normal.

Case 2

While working with TCE within a confined space, this factory assembler developed vertigo, tinnitus, nausea, vomiting, left facial numbness and gait ataxia. Influenza was suspected. Fatigue, tinnitus, hearing loss, loss of tongue sensation, short term memory failure, and inattention persisted for 5 years. MS was suspected by a neurologist and he was referred to the MS Clinic. On examination, horizontal gaze nystagmus, left pinna hypoalgesia, left sensorineural hearing loss, vibratory loss at the toes, lower limb hyperreflexia and absent abdominal reflexes were noted. The auditory evoked potential was absent from the left ear, the left visual evoked response was prolonged (latency $130 \mathrm{msec}$ ), and the cerebrospinal fluid and MRI scan were normal.

TCE is an industrial solvent which was once widely used as an anesthetic for dental and obstetrical procedures. Significant side effects resulted in its discontinuation as an anesthetic agent and has led to the application of rigid guidelines to ensure its safe use in the workplace. ${ }^{\prime}$ The side effects associated with exposure to levels above $100 \mathrm{ppm}$ include restlessness, impaired concentration, irritability, euphoria, bronchial constriction, pulmonary edema, fatal cardiac arrhythmia, and irreversible renal and hepatic damage. ${ }^{1}$

TCE is neurotoxic. Cranial neuropathy may develop within 8-48 hours after exposure. Recovery within 18 months may occur with mild cases but permanent neuropathy is common. Trigeminal neuropathy is particularly common and is frequently bilateral. The recognition that trigeminal sensory neuropathy often followed exposure to anesthesia with TCE during anesthesia resulted in its use as a treatment for refractory trigeminal neuralgia. Dysfunction of cranial nerves II and VII occurs less frequently. Oculomotor disturbances, neuropathies of cranial nerves VIII, IX, X and XII, sensorimotor peripheral neuropathy, tremor, cerebellar, extrapyramidal abnormalities, neurobehavioural changes and coma occasionally result from TCE exposure. ${ }^{2-5}$

The diagnosis of TCE poisoning is suggested by the characteristic history of industrial exposure and by the clinical features outlined above. Following acute inhalation of TCE, the first patient described the immediate onset of systemic symptoms and within $24-48$ hours developed subjective symptoms suggesting a mild trigeminal sensory neuropathy and sensory radiculopathy. The second patient suffered more prolonged and perhaps chronic exposure to TCE. In addition to the symptoms of vertigo, tinnitus, nausea and vomiting which occurred within moments of inhalation, his findings suggested dysfunction of multiple cranial nerves (II, V, VIII) and a myelopathy (vibration loss, hyperreflexia, absent abdominal reflexes).

The mechanism of toxicity is only partially understood. A decomposition product of TCE, dichloracetylene, may be the neurotoxic agent. TCE depresses synaptic transmission and has important effects at the myoneural junction. ${ }^{6}$ TCE may result in demyelination because of its lipid solvent properties. ${ }^{\prime}$ Axonal damage and demyelination in the trigeminal sensory root and descending tract of the trigeminal nerve and neuronal loss in the main and spinal trigeminal nuclei have been reported at autopsy. ${ }^{2-3}$ Other than restricting exposure to TCE, there is no known treatment.

These cases were instructive because they presented with recurrent and chronic fluctuating neurological symptoms and signs which led to the diagnosis of MS by neurologists. In neither case was it possible to diagnose MS on clinical grounds, nor with laboratory investigations. Recognition of this syndrome led to an investigation of the workplace which improved industrial safety.

Neurological syndromes resulting from exposure to toxic chemicals rarely masquerade as MS. Fifty-five of the last 500 new patients (11\%) referred to the University Hospital MS Clinic between 1982-1985 with a diagnosis of MS were found to have an alternate explanation for their neurologic syndrome. Exposure to toxic chemicals was not a factor in any of these patients. We are unaware of another case of industrial exposure to TCE presenting as MS in our 1600 patient clinic population or in the literature, but other cases may not have been recognized.

$$
\begin{array}{r}
\text { John H. Noseworthy } \\
\text { George P.A. Rice } \\
\text { University of Western Ontario } \\
\text { London, Ontario }
\end{array}
$$

\section{REFERENCES}

1. Feldman RG. Trichlorethylene. In: Vinken PJ and Bruyn GW, eds. Handbook of Clinical Neurology. North Holland 1979, Vol 36: 457.464 .

2. Humphrey $\mathrm{JH}$ and $\mathrm{McClelland} \mathrm{M}$. Cranial nerve palsies with herpes following general anesthesia. Br Med J 1944; 1: 315-318. 
3. Buxton PH and Hayward M. Polyneuritis cranialis associated with industrial trichloroethylene poisoning. J Neurol Neurosurg Psychiatry 1967; 30: $511-518$

4. Lawrence WH and Partyka EK. Chronic dysphagia and trigeminal anesthesia after trichloroethylene exposure. Ann Intern Med 1981; 95: 710 .

5. Lindstrom K. Behavioural effects of long-term exposure to organic solvents. Acta Neurol Scand 1982; 66: 131-141.

6. Kennedy RD and Galindo AD. Comparative site of action of various anesthetic agents at the mammalian myoneural junction. $\mathrm{Br} \mathrm{J}$ Anaesth 1975; 47: 533-540.

\section{SPONTANEOUS TEMPORARY REMISSION IN PRIMARY CNS LYMPHOMA}

\section{To the Editor}

In regard to the article by Rubin et al,' we would like to add that the diagnosis of primary CNS lymphoma appears to be much more common recently than ever in the past, in patients who are not infected with HIV. We have also noted temporary remission, but on careful, detailed, methodical review of patient information, these remissions correlate with some type of corticosteroid medication having been given. In one case, for example, this corticosteroid medication was given as part of an angiographic procedure and was even omitted from the medication record. It is therefore our hypothesis that routine doses of corticosteroid medication are potentially capable of inducing transient remissions. Such temporary remissions can be of serious consequence if physicians mistakenly assume that "all is well" and do not proceed to biopsy and radiation treatment. This is particularly important now, with the availability of computer-assisted stereotactic biopsy techniques minimizing morbidity.

It would be of interest to know if the clinical record of the case reported by Rubin et al, was exhaustively scrutinized for any type of corticosteroid medication given at the time of her initial evaluation.

Walter F. Buell Robert G. Hart University of Texas San Antonio, Texas

\section{REFERENCES}

1. Spontaneous temporary remission in primary CNS lymphoma. Can J Neurol Sci 1987; 14: 175-177.

\section{REPLY}

We thank Drs. Buell and Hart for their comments. Although our patient did undergo angiography, at no time during her first admission did she receive any steroid medication. Careful review of the chart and questioning of the house staff verified this. Nonetheless, remission following routine doses of steroid medication given as part of an angiographic procedure is equally surprising. Either it was purely coincidental or, based on proposed mechanisms of steroid-induced remission, the level of gluco-corticoid receptor in the cytosol was extremely high.

With regard to the frequency of primary CNS lymphoma in non-HIV infected patients, our review of the literature indicates that this entity, even in the past, was only rarely seen in those immunocompromised for any reason. ${ }^{1,2,3}$ Of 400 cases reported, only $1 \%$ were immunocompromised. ${ }^{4}$ Thus primary CNS lymphoma has always been much more common in persons immunologically intact.

\author{
Michael Rubin \\ Israel Libman \\ College of Physicians \& \\ Surgeons of Columbia \\ New York, N.Y.
}

\section{REFERENCES}

1. Barnard RO, Scott T. Patterns of proliferation in cerebral lymphoreticular tumors. Acta Neuropathol 1975; 6: 125-130.

2. Enzmann DR, Krikorian J, Norman D, et al. Computed tomography in primary reticulum cell sarcoma of the brain. Radiology 1979; 130: $165-170$.

3. Henry JM, Heffner RR Jr., Dillar SH, et al. Primary malignant lymphomas of the central nervous system. Cancer 1974; 34: 1293-1302.

4. Helle TL, Britt RH, Colby TV. Primary lymphoma of the central nervous system. Clinicopathological study of experience at Stanford. J Neurosurg 1984; 60: 94-103. 